\title{
82. Wann erschienen die Poronai-Faunen zum erstenmal?
}

Von Masao Minato, Masaru Matsui, Satoru Uozumi, Yasukuni Furuhata, Tsutomu Fujie, und Ichiro Hayashi.

(Comm. by H. YABE, M.J.A., July 12, 1952.)

Es war Yabe selbst, der zum erstenmal vor beinahe einem halben Jahrhundert die Bemerkung gemacht hat, dass eine bedeutende Diskordanz unterhalb der Poronai-Formation bestehe. Und seit Yabe bezweifelte niemand, dass die dortige stratigraphische Beziehung nichts anderes als Diskordanz sei.

In letzter Zeit aber betrachtet Yabe die Art der stratigraphischen Beziehung zwischen Poronai- und Ishikari-Formation als unentscheidbar. Die sogenannte Ishikari-Serie schliesst den Teil, der die Meer-Fazies darstellt, relativ wenig ein, ausser der WakkanabeFormation, welche Faunen von der jenigen der Poronai-Formation etwas verschieden sind. Trotzem gibt es sicher einen solchen Teil wie Meer-bzw. polyhalinische-Fazies hie und da in den Schichten oberhalb der Wakkanabe-Formation; besonders treten die Ostreabzw. Corbicula Schalenzonen in der Oberen bzw. Unteren CorbiculaFormation reichlich auf. Solche Fazies zeigt zweifelsohne, dass damals ein Meer unweit der Ablagerungsbezirke der Ishikari-Serie vorhanden war.

Tabelle I

Die Formationsreihe

auf der westlichen Seite des auf der östlichen Seite Hidaka-Gebirges

Poronai F.

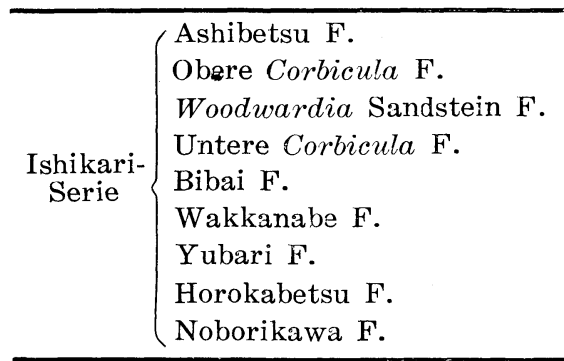

Liegendes: Kreide

$$
\text { des Hidaka-Gebirges }
$$

Heutzutage ist Yabe $^{1)}$ der Ansicht, dass die Poronai Formation von einen solchen Meere mitgebracht worden sei, und deshalb nicht

1) H. Yabe: Stratigraphical relation of the Poronai-and Ishikari-Groups in the Ishikari Coalfield, Hokkaido, Proc. Japan Acad., 27, 571 (1951). 
nur auf der Ishikari-Serie liege, sonderen auch zum grössten Teil stratigraphisch zu diese in Parallele stehe: kurz gesagt, die PoronaiFormation sei meistens gleichzeitig mit der Ishikari-Serie und zum Teil nach der Ashibetsu-Zeit abgelagert worden.

Dabei hat Yabe, um seinen Ansicht zu rechtfertigen, einige palaeontolngischen Tatsachen angeführt, dass die gleichen Spezies wie bei der Poronai-Formation auch in der Ishikari-Serie, vorkommen, z.B. Yoldia bzw. Nuculana sowohl in der Poronai-Formation als auch in der Ishikari-Serie (Obere Corbicula-Formation).

Man glaubt im allgemeinen, dass die Uraforo-Serie, die auf der östlichen Seite des Hidaka-Gebirges weit verbreitet ist, dem oberen Teil der Ishikari-Serie stratigraphisch beinahe vergleichbar ist.

Nun ist Cornuspiroides oinomikadoi Hanzawa et Asano sowohl aus der Poronai-Formation als auch aus der Uraforo-Serie (Shitakara-Formation) bekannt.

Ferner hat Yabe auf die Tatsache aufmerksam gemacht, dass die eozäne Foraminifera Cyclamina pacifica Beck aus der PoronaiFormation von Asano gesammelt worden ist.

Neuerdinges ist Linthia yessoensis Minato ${ }^{2)}$ zufällig auch aus dem unteren Teile der Shitakara-Formation in der Gegend von Yubetsu, auf der östlichen Seite des Hidaka-Gebirges bekannt geworden.* Diese Spezies war bekannt aus der Formation oberhalb der Woodwardia-Sandstein-Formation in der Ishikari-Serie ${ }^{3)}$, auf der westlichen Seite des Hidaka-Gebirges, und deshalb ist die Möglichkeit sehr gross, dass der Horizont der Linthia yessoensis der Oberen Corbicula-Formation angehört.

Daher glauben wir jetzt dass die Shitakara-Formation mit der Corbicula-Formation stratigraphisch vergleichbar ist.

Wie schon bereits bekannt, treten viele polyhalinische bzw. Meeres Molluskenfossilien aus der Shitakara-Formation reichlich auf, darunter: Yoldia asagaiensis Mak., Y. laudabilis Yok., $Y$. watasei Kan., Nuculana sp., Thyasira bisecta (Conrad), Macoma ef. dissimilis Mart., Periploma besshoensis (Yok.), Nemocardium yokoyamai Tak., N. ezoensis Tak., Venericardia akagii Kan., Chlamys kushiroensis Uoz., Mya grewingki Mak., Mya grewingki var. kushiroensis Nag. et Inoue., Neptunea shitakaraensis Mat., Olivella cf. iwakiensis Nom. et Hat.

2) M. Minato: On some Palaeogene Foss:is in Hokkaido. Jour. Geol. Soc. Japan, Vol. 56, No. 655, 1950.

3) M. Mita and T. Kojima: Preliminary notes for the Geology in the vicinity of the Bannosawa, Upper Tributary of the River Penke-Poronai, Ashibetsu-machi, Bull. Geol. Surv. Japan, Vol. 1, No. 2, 1951.

* Wir glauben dass Linthia yessoensis aus der Oberen Corbicula-Formation kommt, obwohl Mita und Kojima die Horizonte von Lithia yessoensis nur als Schichten oberhalb der Woodwardia-Sandstein-Formation bezeichnet haben. 
Tabelle II

Fossilinhalt der Yubetsu-, Shitakara- und Onbetsu-Formation

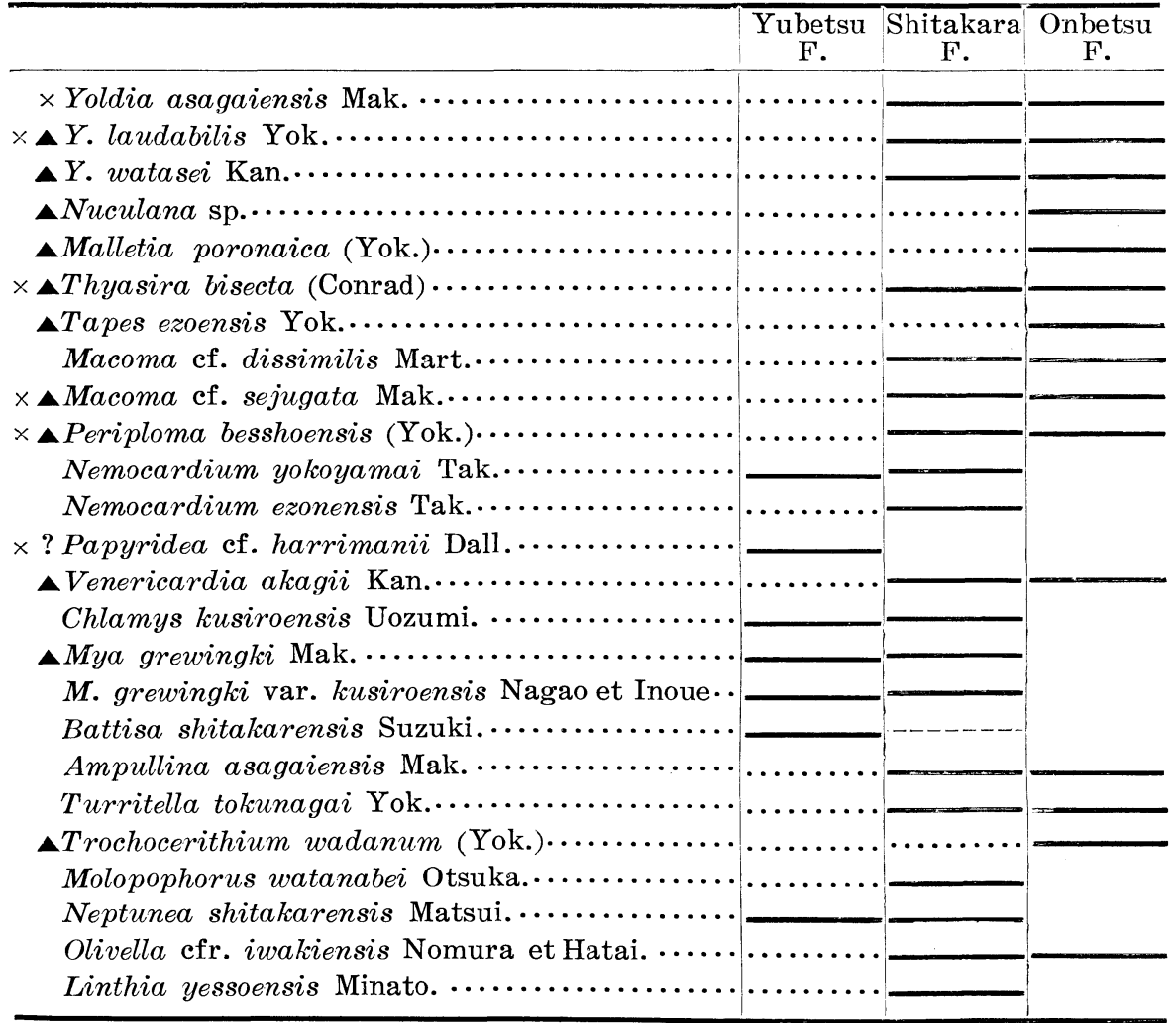

$\times$ aus der Asagai-Formation bekannt

- aus der Poronai-Formation bekannt

Nicht wenige Arten unter den oben erwähnten Spezies sind auch aus der Poronai-Formation bekannt, und deshalb kann man nicht verneinen, dass die Poronai-Faunen wenigstens zum Teil anfangs in der Oberen Corbicula Zeit erschienen sind.

Nun aber hat gerade vor einem halben Jahre einer der Verf. (Hayashi) zufällig in der Gegend östlich von Kushiro im unteren Teile der Yubetsu-Formation einige Mollusken-Fossilien entdeckt, was besonders wichtig ist, da bisher keine Meer-Arten aus diesem Horizont bekannt waren.

Bis jetzt handelt es sich um folgende Spezies: Nemocardium yokoyamai Tak., Papyridea sp., aff. harrimanii Dall, Chlamys kushiroensis Uoz., Mya grewingki Mak., M. grewingki var. kushiroensis Nag. et Inoue, und Neptunea shitakaraensis Mat.

Darunter ist Chlamys kushiroensis eine Spezies, die auch zu den Exemplaren der obigen Liste der Shitakara-Formation gehört. Nemocardium yokoyamai Tak., Mya grewingki Mak., M. grewingki 
var. kushiroensis und Nep. shitakaraensis kommen auch in dieser Shitakara-Formation vor; dagegen ist Papyridea ef. harrimanni Dall ebenfalls beachtenswert für diesen Horizont, die in der AsagaiFormation beim Joban-Kohlengebiet besonders reichlich auftritt.

Daraus können wir schliessen, dass die Poronai-Faunen, wenigstens zum Teil, schon in der Yubetsu-Zeit erschienen sind.

Diese Zeit entspricht beinahe der Zeit des oberen Teiles der Woodwardia-Formation bzw. des unteren Teiles der Oberen CorbiculaFormation auf der westlichen Seite des Hidaka-Gebirges.

Die Sache erklärt sich in der Weise, wie oben erwähnt d.h. niemand kann mehr die Möglichkeit verneinen, dass die PoronaiFormation zum Teil mit der Ishikari-Serie in Parallele steht.

Wenn die stratigraphische Beziehung zwischen der IshikariSerie und Poronai-Formation eine Diskordanz sein sollte, wie man lange geglaubt hat, mag jedenfalls die stratigraphische Lücke dafür zeitlich nicht bedeutend sein.

Zum Schluss fühlen wir die Pflicht, unserem verehrten Lehrer, Herrn Prof. I. Hayasaka, für seine gütige Anleitung zu danken. Wir möchten ausserdem es nicht unterlassen, an dieser Stelle den Herren H. Yabe, S. Tashiro, M. Nagabuchi, S. Takao, S. Mabuchi, M. Hosono, S. Nishida, K. Sugai, F. Takai, S. Mita, und K. Asano für ihre vielfachen und stets hilfsbereiten Anregungen zu danken. 